QL

435

.A1

086

1891

INVZ 
is conse

ricerise

incelese

merar

is
तद (द⿱

ciare

7

$2 \pi$

है

(I)

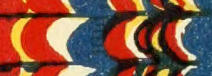

121

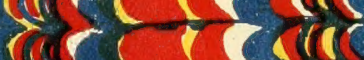

(G) 121$)^{\circ}$

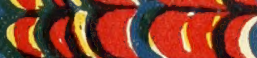

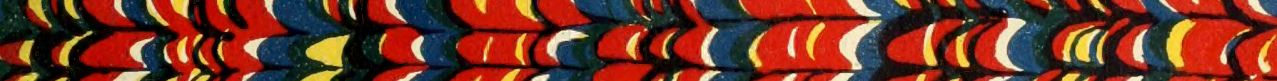

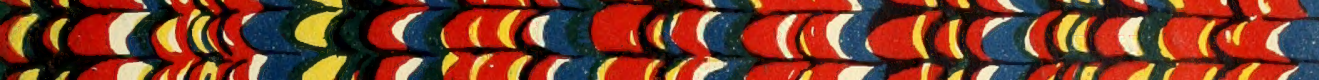

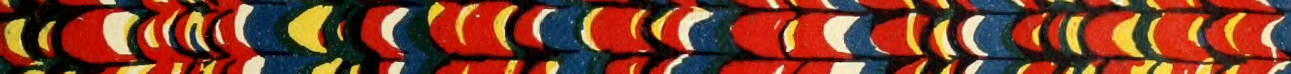

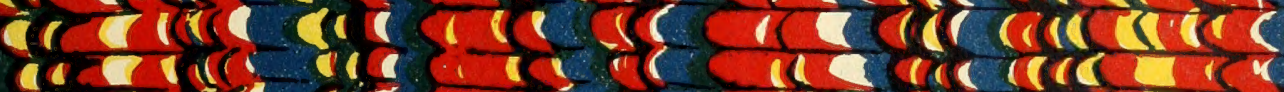

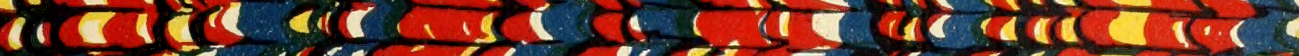

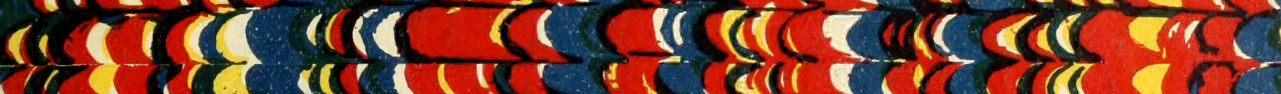

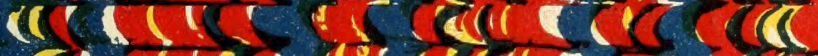

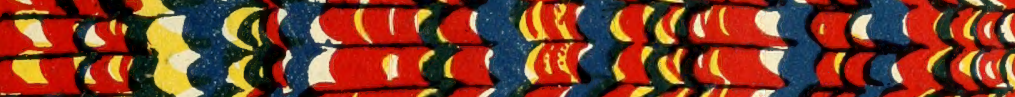

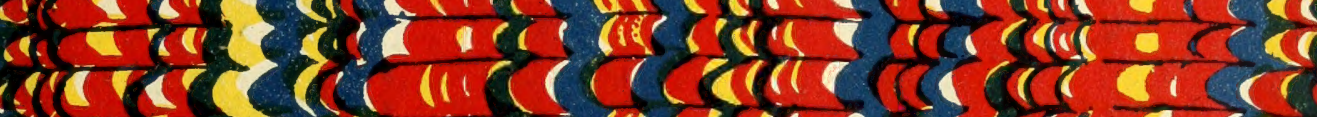

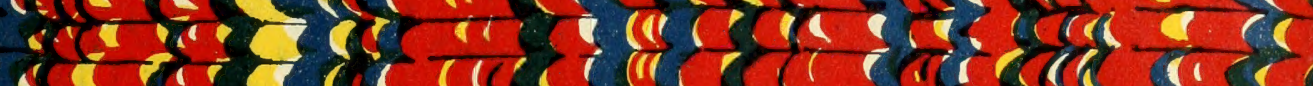
c.

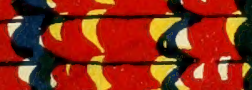
Cis 19 हो सिखि $7,1,0,1$ i 1 T) C. PTic $1 \mathrm{CaC}$ $\mathrm{Cr} \mathrm{COC}$ $1128+2$

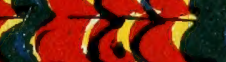
?.

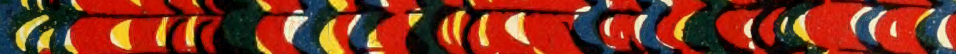

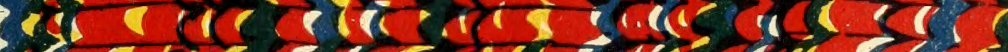
$a$
$a$

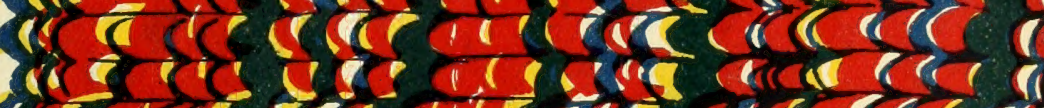

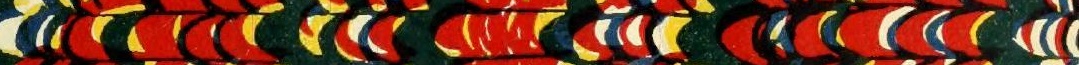

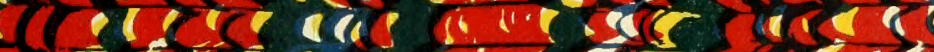

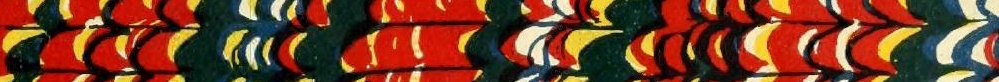

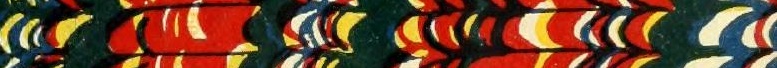

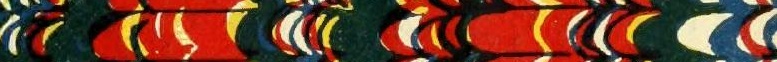
$\sin \operatorname{sic}$

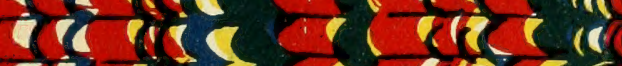

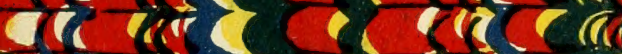
ir ris? cरेखित ir $r(c$

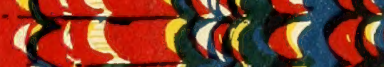

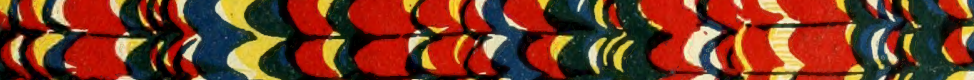

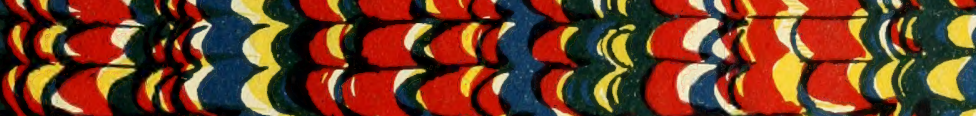

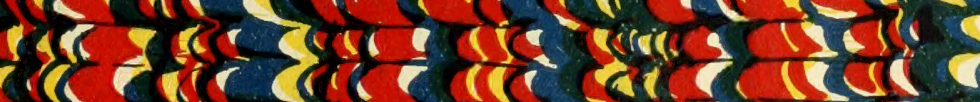

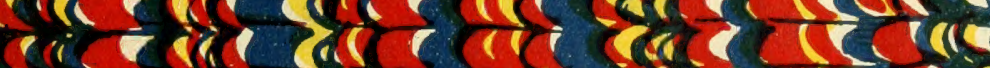

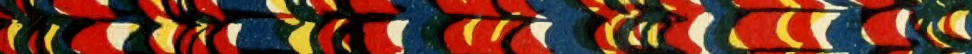

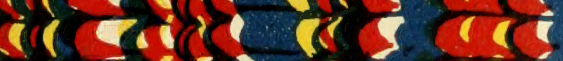
c)

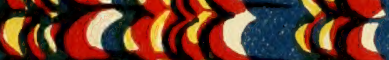
ace 10 sias sesces (ii) ? $\operatorname{loc} C \operatorname{cc}$ (1) 149 in $\rightarrow$ $\frac{1}{1}=\frac{\pi}{\operatorname{sic} t}$ () 


Osorio, Bathazar Oristacea. (Binder's title).

244747 

Osiris

595.3

$6+922$
Som the

63 





1.

i 



\title{
APPENDICE AO CATALOGO DOS CRUSTACEOS DE PORTUGAL EXISTENTES NO MUSEU NACIONAL DE LISBOA
}

\author{
Pori \\ BALTHAZAR OŹORIO
}

O appondice que hoje se publica comprehende não só um numero relativamente subido de novas especies a juntar á lista dos crustacens de Portugal, uma das quaes é além d'isto, segundo cremos, inteiramente ignorada dos carcinologistas, mas tambem n'elle se enumeram outras já conhecidas, cujo habitat se accrescenta.

Juntamos ainda, a algumas d'estas ultimas especies, notas sobrc caracteres que observámos, sobre a profundidade a que se eneontram c sobre a epocha em que se dá a evolução. As observações sobre este ultimo assumpto parecem-nos importantes, pois devem interessar á industria das peseas e ao legislador, a quem importa conhecer, por mais de um titulo, a epucha da reproducção das especies que stio enmestiveis do homem ou dos.peixes.

\section{Stenorhynchus phalangium. Penn.}

(a) N. de Sines (S. A. R. o Senhor Infante D. Affonso).

(b) Nas redes Charrão a $1^{\mathrm{m}}$ da maxima baixa-mar.-Muitas femeas com os ovos, colhidas em junho.

(c) Um indivivuo novo colhido entre 15 a 30 metros na barra do Sado (sr. A. Girard).

2. Inachus scorpio, Fabr.

Foz do Douro (sr. Isaac Newton).

3. Lambrus setubalensis, Capello. Affonso).

$\mathbf{N}$. de Sines, 36 bxaças de profundidade (S. A. R. "Infinte D. JORN. DY SCIINC, MATHI. PHYS. E NAT. $20^{2}$ SERIE-N. VIII. 
4. Cancer pagurus, Linn.

Exemplar de grandes dimensões.

Colhido pelos vapores de pesca entre o Porto e Espozende (sr. Isaac Newton).

5. Xantho rivulosus, Risso.

¡ com os ovos. Colhida nas poças d'agua no maximo baixa-mar, em Setubal, junho (sr. A. Girard).

6. Pilumnus hirtellus, Penn.

ふ. Foz do Douro (sr. Isaac Newton).

7. Eriphia spinifrons, Herbst.

§. Setubal (sr. A. Girard).

Todos os exemplares d'esta especie que existem no Museu teem uma côr amarellada, emquanto que o exemplar colhido em Setubal tem a côr vermelha vinosa que Milne Edwards lhe assignala.

8. Portunus arcuatus, Leach.

q com os ovos. Junho. Setubal (sr. A. Girard).

9. Carcinus maenas, Penn.

(a) Porto.-(b) Rio Douro, ô (sr. Isaac Newton).

10. Gonoplax angulata, Fabr.

f ๙. Porto (sr. Isaac Newton).

Dos seis exemplares que observámos só um tem os espinhos postorbitarios. O espinho do braço não está ao meio como diz MI. Edwards, nem sempre quasi ao meio, como diz Bell, mas na união do primeiro terço com o segundo.

11. Pachygrapsus marmoratus, Rondelet.

Cascaes.

12. Ebalia Pennantii, Leach.

ๆ ช. Cezimbra (sr. A. Girard).

Nas redes Charrão $1^{\mathrm{m}}$ abaixo da maxima baixa-mar. Femeas com os ovos colhidas em junho. 
13. Calappa granulata, Fabr.

(a) q. Porto (sr. Isaac Newton).-(b) \$. Colhido pelos vapores de pesea entre o Porto e Espozende (sr. Isaac Newton).

14. Dorippe lanata, Bosc.

ふ. Alfeite (sr. J. A. de Sousa).

15. Eupagurus Bernhardus, Linn.

Foz do Douro (sr. Isaac Newton).

16. Pagurus striatus, Latr.

Colhidos pelos vapores de pesca entre o Porto e Espozende (sr. Isaac Newton).

17. Pagurus Hyndmanni, Thomps.

Barra do Sado. Dragagem entre 15 e $20^{\mathrm{m}}$. $q$ com os ovos. Junho (sr. A. Girard).

Esta especie já tinha sido incluida no nosso catalogo, porém sem indicaçào de hubitat. $O$ exemplar que n'elle mencionavamos tinha-nos sido enviarlo pelo sr. dr. Paulino e tinha sido colhido tambem em Setubal.

18. Porcellana longicornis, Penn.

N. de Sines. 34 braças de profundidade (S. A. R. o Senhor Infante D. Affonso).

19. Galathea squamifera, Leach.

(juv.) Costa de Cezimbra (sr. A. Girard).

20. Scyllarus arctus, Linn.

Costa de Portugal. Duas $q$ com os ovos colhidas em julho (st. Victor Sassetti).

Sabemos que esta especie tem sido colhida no Algarve, pois vimos exemplares d'esta procedencia.

21. Nephrops norwegicus, Linn.

Golhido pelos vapores de pesseal entre o Porto e Espozende (sr. Isaac Newton). 
22. Grangon vulgaris, Fabr.

Setubal. Nas redes Charrão, $1^{\mathrm{m}}$ abaixo da maxima baixa-mar. Exemplares com os ovos, collidos em maio e jumho (sr. A. Girard).

23. Palaemon squilla, Linn.

24. Talitrus locusta, Linn.

(a) Cascaes.-(b) Praia O. de Setubal. Debaixo dos limos na maré cheia (sr. A. Girard).

25. Armadillo officinarum, Brandt.

Alcolena (sr. Manuel de Sousa).

26. Armadillium vulgare, Latr.

Alcolena (sr. Manuel de Sousa).

27. Anilocra physodes, Iinn.

No nosso Cataloyo dos crustaceos de Portugal já tinhamos apontado esta especie, que reconhecemos com outros carcinologistas, Carus, por exemplo, ser perfeitamente identica á $A$. mediterrunea, sob cuja designaçào a tinhamos inscripto. Nào sabiamos, porém, onde tinham sido encontrados os exemplares que existiam no Museu. Ultimamente recebemol-os de Setubal onde foram colhidos pelo sr. A. Girard. Parece que é parasita da Corvina, Sciaena aquila, Cuv. et Val. Os exemplares colhidos em junho teem debaixo das laminas ineubadoras um grande numero de ovos ou individuos d'esta mesma especie, tendo apenas um a dois millimetros de conprimento, em grandissimo numero, destacando-se perfeitamente n estes os olhos, pela sua côr extremamente escura e pela sua grandeza relativa desproporcionada.

As especies a accrescentar ao Catalogo são as seguintes:

1. Pilumnus spinifer, Edw.

M. Edw., Hist. nat. des crustacís, t. I, p. 420; Heller, Crrust. Südlich. Eurp., p. 73 .

ô o com os ovos.

Setubal ou Cezimbra (sr. A. Girard).

2. Atelecyclus heterodon, Leach.

Malac. Brit., tab. II ; M. Edw., Hist. nat. des Crust., t. II, pag. 143; Bell. British. Crust., p. 153; Heller, Crust. Siidlich. Europ., p. 133.

$\delta$ (juv.) N. de Sines. 36 braças de profundidade (S. A. R. o Senhor Infante D. Affonso). 


\section{Crangon fasciatus, Risso.}

Crust. de Nice, p. 82, pl. III, fig. 5; M. Edw., loc. cit., t. 11, p. 342 ; Bell. Brit. Crust., p. 259; Heller, loc. cit., p. 228, tab. VII, fig. 10.

Setubal. Nas poças d'agua na maxima baixa-mar (sr. A. Girard).

\section{Genus ALPHEUS}

4. Alpheus platyrhynchus, Heller.

A. Edwardsii, M. Edw., loc. cit., t. II, p. 352; A. platyrhynchus, Heller, loc. cit., p. 276, tab. IX, fig. 18 e 19.

Barra de Lisboa (S. A. R. o Senhor Infante D. Affonso).

\section{Genus VIRBIUS, Stimps.}

5. Virbius viridis, Otto.

Alpluens viridis, -Nov. acta Acad. Leop. Carol, t. xıv, tab. XX, fig. 4 ; Hyppolite viridis, M. Edw., loc. cit. t. II, 372; Virbius viridis, Heller, loc. cit., p. 286, tab. X, fig. 3; Carus, Faun. Mediter., t. n, p. 478.

Côr verde-mar. Setubal. Na rede Charrĩo, $1^{\mathrm{m}}$ abaixo da maxima baixa-mar, $q$ com os ovos, junho (sr. A. Girard).

Genus ORCHESTIA, Leach.

6. Orchestia littorea, Leach.

Fidinl. Encyc., vir, pl. CCXXI, fig. 6; M. Edrv., loc. cit., t. IIr, p. 16; Sp. Bate, Catal. of Amphip. Crust., p. 27, tab. IV, fig. 8.

Setubal. Debaixo dos limos (sr. A. Girard).

7. Orchestia Deshayesii, Audouin.

Savigny, Egypte, Crust., pl. XI, fig. 8; Sp. Bate. loc. cit., p. 23., pl. IV, fig. 3.

Setubal. Debaixo dos limos na maré cheia, praia oeste (sr. A. Girard).

Spence Bate diz que conhece apenas um numero muito limitado de exemplares d'esta especie. 
Genus IDOTEA, Fabr.

8. Idotea appendiculata, Risso.

Leptosoma appendiculata-Hist. nat. de l'Eur. mírid., t. v, p. 107, tab. IV, fig. 23; Idotea appendiculata, - Hist. nat. des Crust., t. II, p. 135; Carus, Faun. Méditer., t, II, p. 449.

(a) Cascaes.-(b) Foz do Douro (sr. Isaac Newton).-- (c) Granja (sr. dr. Paulino).

Esta especie era conhecida, antes do nosso estudo, do illustre professor da Úniversidade cujo nome acabamos de mencionar.

9. Idotea acuminata, White.

Carus, loc. cit., t. II. p. 449.

Foz do Douro (sr. Isaac Newton).

Um exemplar sem as manchas orladas de negro mencionadas pelos carcinologistas.

10. Idotea tricuspidata, Desm.

Consider., p. 289; M. Edw., loc. cit., t. III, p. 129; Carus, loc. cit., t. 1 , p. 448.

Foz do Douro (sr. Isaac Newton).

\section{Idotea hectica, Latr.}

Hist. nat. des Crust., t. vi, p. 371; Lamarck, Hist. nat. des anim. sans vert., t. v, p. 269; Idotca viridissima, Risso, Crust. de Nice, p. 136, pl. III, fig. 8; Idotea hectica, M. Edw., loc. cit.. t. II, p. 133 ; Carus, loc. cit., t. II, p. 448.

Setubal. Sobre os limos, $1^{\mathrm{m}}, 20$ abaixo da maxima baixa-mar, na rede Charrão. Verde-claro (sr. A. Girard).

\section{Genus LYGIA, M. Edw.}

12. Lygia oceanica, Fabr.

Supplem., p. 301 ; M. Edw., loc. cit., t. III, p. 155; Carus, loc. cit., t. IJ, p. 455.

Praia do Caramujo (sr. A. Girard).

Esta especie tinha-nos sido enviada pelo sr. dr. Paulino que a tinha colhido em Aveiro, antes de termos obtido os exemplares da procedencia que designamos acima. 


\title{
FAM. BOPYRIDAE, LATR.
}

\author{
Genus BOPYRUS, Lat.
}

13. Bopyrus squillarum, Latr.

Hist. nat. des Crust. vir, p. 55, pl. LIX, fig. 2, 4; Lamarck, Hist. nat. des anim. sans vert., t. v, p. 164; M. Edw., loc. cit., t. III, p. 282; Carus, loc. cit., t. Ir, p. 452 .

Na camara branchial d'um exemplar da Nitia edulis que foi colhido em Setubal. Conheciamos já esta especie por nos ter sido enviada pelo sr. dr. Paulino que a havia colhido, ba alguns annos, em Villa Nova de Mil Fontes, não sabemos porém sobre que especie.

Genus ROCINELA, Leach.

14. Rocinela Desaysiana, M. Edw.

Hist. nat. des Crust., t. III, p. 243.

Praia de Setubal (sr. A. Girard).

\section{Genus ARGULUS, O. F. Müller}

Sub-genus Agenor, Thor.

15. Agenor purpureus, Thor.

Binoculus cornutus, Risso, loc. cit., p. 170; Carus, loc. cit., p. 290.

Parasita do Trachurus trachurus, L.

Mercado de Lisboa (sr. Manuel de Sousa).

Mosca do mar. Nome dado pelos pescadores.

Genus PANDARUS, Leach.

16. Pandarus bicolor, Leach.

Encyc. Brit. supplem., t. I, pl. XX; Latr. Encyc. method., pl. CCCXXXI, fig. 25,26 ; M. Edw., loc. cit.. t. IIr, p. 470 ; Carus, loc. cit., t. II, p. 362.

White diz que esta especie se encontra sobre o Mustelus vul.garis. 
Os nossos exemplares foram colhidos n'um individuo do genero Mlustelus, mas não sabemos em que especie.

Setubal (sr. A. Girard).

Genus LERNAEA, Linn.

\section{Lernaea branchialis, Linn.}

Syst. nat., Guerin, Iconogr. Zooph., pl. IX, fig. 1; M. Edw., loc. cit., t. III, p. 528 ; Carus, loc. cit., t. II, p. 371.

Parasitas vivendo no apparelho branchial de un Merlucius vulgaris, Flem. Carus diz que esta especie vive nos arcos branchiacs do Merlucius esculentus, Risso, que é considerado por Gunther como identico ao $M$. vulgaris.

(Sr. A. Girard).

Genus PERODERMA, Rich.

18. Peroderma Capelloi, n. sp.

Espèce très voisine du $P$. cylindricum, Heller, (Tiqphrobia pilchurdus, Cornalia) et comme ce parasite vivant logé aux muscles du $C$. pilchardus, Walb.

L'ouverture de ee logement, qui monte obliquement dans les tissus, se trouve un peu au dessus de l'extrémité de la nageoire pectorale. Il est vraiment remarquable que le parasite dans nos einq individus aussi bien qu'au exemplaire de Cornalia ${ }^{1}$ s'est introcuit dans le corps de l'animal par le côté droit.

La longueur du corps de notre espèce est plus courte que celle du $P$. cylindricum, mésurant 13 millimètres dans le plus grand de nos individus, 1 cent. dans le plus petit et non 14 millim. (Cornalia) ou 15 millim. (Carus) ${ }^{2}$

L'extrémité postérieure se termine par trois lobes, le médian bilobé. Ce caractère distinguerà aussi notre espèce de celle d'Heller.

Les ovaires, qui suivant Cornalia ont un millimètre de largeur, ont dans nos individus seulement $1 / 2$ millim.

Nos exemplaires présentent des différences notables au point de vu $d u$ développement des ovaires; seulement un seul ovaire est visible, on tous les deux à la fois, un plus long que l'autre, mais il arrive aussi que les deux n'existent pas. Ont-ils tombés ou sont encore à paraitre? Je ne le sais dire. Chez les individus ainsi conformés la partie

${ }^{1}$ Sulle Taphrobia pilchardus - Nuovo genere do crostrci parassiti-note del prof. E. Cornalia.

${ }_{2}$ Prodr. Faun. Médit., q. Ir, p. 373. 
senlement visible an dehors c'est l'extrémité postérieure trilobé. (Shez les individus à ovaires bien développés, ceux-ci mésurent 5 centimètres, longueur totale du exemplaire de Cornalia.

Il y a un caractère qui aidera distinguer tout de suite notre espèce, c'est la conformation du cou ou canal suceur lateral, puis qu'il se compose de diverses ramifications, terminées par des grossissements, qui lui donnent de la ressemblance avec une toute petite chou-fleur. Ces grossissements me semblerent traversés au milieu par un camal, mais dans une préparation que j'ai fait pour m'assurer de cette circonstance je n'ai pu arriver à une conviction, parcequ'il est très difficile de separer des tissus le canal suceur sans l'endommager, tant son adhésion à ceux ci est intime.

Cette espèce récueillie par F. Capello dans une de ses explorations zoologiques à Setubal, quand son intelligence eornmenȩait déjì à baisser, est resté depuis lor's oublice dans les vitrines du NiInséum. Je la consacre à la mémoire de ce travailleur vaincu, de ce vaillant pionnier de la science contemporaine. 







$m q / 525188$

Prease ado to Picicust 